Note: This is a preprint of a paper being submitted for publication. Contents of this paper should not be quoted nor referred to without permission of the author(s).

To be submitted to 95 Fall MRS Proceedings

\title{
Stark Effects on Band Gap and Surface Phonons of Semiconductor Quantum Dots in Dielectric Hosts
}

\author{
R. Mu, A. Ueda, Y-S. Tung, and D. O. Henderson \\ Fisk University \\ Nashville, TN \\ J. G. Zhu, J. D. Budai, and C. W. White \\ Oak Ridge National Laboratory \\ Oak Ridge, TN
}

\author{
"The submitted manuscript has been \\ authored by a contractor of the U.S. \\ Govermment under contract No. DE-ACO5- \\ 960R22464. Accordingly, the U.S. \\ Government retains a nonexclusive, \\ royalty-free license to publish or \\ reproduce the published form of this \\ contribution, or allow others to do so, for \\ U.S. Govemment purposes." \\ Prepared by the \\ Oak Ridge National Laboratory \\ Oak Ridge, Tennessee 37831 \\ managed by \\ LOCKHEED MARTIN ENERGY RESEARCH CORP. \\ for the \\ U.S. DEPARTMENT OF ENERGY \\ under contract DE-AC05-96OR22464
}

\section{January 1996}

DISTRIBUTION OF THIS DOCUMENT IS UNLIMITED

\section{DISCLAIMER}

This report was prepared as an account of work sponsored by an agency of the United States Government. Neither the United States Government nor any agency thereof, nor any of their employees, makes any warranty, express or implied, or assumes any legal liability or responsibility for the accuracy, completeness, or usefulness of any information, apparatus, product, or process disclosed, or represents that its use would not infringe privately owned rights. Reference herein to any specific commercial product, process, or service by trade name, trademark, manufacturer, or otherwise does not necessarily constitute or imply its endorsement, recommendation, or favoring by the United States Government or any agency thereof. The views and opinions of authors expressed herein do not necessarily state or reflect those of the United States Government or any agency thereof. 


\title{
STARK EFFECTS ON BAND GAP AND SURFACE PHONONS OF SEMICONDUCTOR QUANTUM DO'TS IN DIELECTRIC HOSTS
}

\author{
R. MU*, A. UEDA*, Y-S. TUNG*, D O. HENDERSON*, JANE G. ZHU**, JOHN D. BUDAI** \\ and W. C. WHITE** \\ ${ }^{*}$ Chemical Physics Laboratory, Department of Physics, Fisk University, Nashville, TN 37208 \\ **Oak Ridge National Laboratory, Oak Ridge, P. O. Box 2008, Oak Ridge, TN 37831-6057
}

\section{ABSTRACT}

We have investigated quantum-confined Stark effect (QCSE) on GaAs and CdSe nanocrystals and the electric field effect on surface phonons of $\mathrm{GaAs}$ nanocrystals isolated in sapphire substrates. For a strongly quantum-confined system, GaAs quantum dots illustrated no exciton energy shift. When the excitons are weakly confined in $\mathrm{CdSe}, \mathrm{a} \sim 2 \mathrm{meV}$ red-shift was observed. On the other hand, the results of the electric field effect on surface phonon are dramatic both phonon oscilator strength and freqnency. As the strength of the electric field increases, the total intensity of the surface phonon decreases. At the same time, an additional peak was also observed at $277 \mathrm{~cm}^{-1}$, which is about $3 \mathrm{~cm}^{-1}$ above the center frequency of the surface phonon mode of GaAs nanocrystals embedded in a sapphire host.

\section{INTRODUCTION}

The study of semiconductor quantum dots (QDs) has attracted much attention in recent years due to the unique optical properties considerably differing from the parent (bulk) materials. To understand the physical and optical properties of the QDs and to fabricate more controllable quantum dots in terms of size and shape in various host materials are both the intriguing and challenging fundamental problems and the crucial steps towards the true realization of the new device applications. There have been number of ways ${ }^{1}$ developed to fabricate quantum dots in different solid host materials and in solutions, such as inorganic synthesis, sol-gel synthesis, impregnation into porous hosts, vapor deposition, and ion implantation. Recently, it has been demonstrated that the sequential ion implantation of II-VI and III-V elements into dielectric hosts followed by thermal annealing can lead to semiconductor quantum dots formation. The combination of linear optical and far-infrared reflectance (FIR) spectroscopy has been proved to be an effective characterization technique to identify the formation of quantum dots in dielectric hosts. The linear spectroscopy can be used to study excitonic absorption energy of the QDs due to the quantum confinements, while FIR technique can be used to study the surface phonon mode of the QDs falling between TO and LO modes of the bulk.

In order to increase the versitility of the optical and vibrational techniques into the size- and shape-sensitive tools in characterizing QDs in various dielectric hosts, efforts have been made to study electric field effects on exciton energy and the surface phonon modes of the QDs embedded in the matrix

\section{THEORETICAL CONSIDERATION}

\section{Quantum-Confined Stark Effect (QCSE) on Quantum Dots}

QCSE on quantum well studied extensively in the past in a series of investigation by Miller at $a l .{ }^{9}$ New devices, such as optical modulators and self-electrooptical devices (SEED) have been developed based on this effect. When the quantum dots are placed in an static electric field $F$, both 
the exciton energy and their oscillator strength are modified. A model calculation by Jaziri ${ }^{4}$ showed that the total energy of the excitons confined in quantum dots will be expected to be red-shifted under the applied electric field. The amount of red-shift is quadratically proportional to the applied electric field. For the GaAs quantum dot with a particle size of $20 \mathrm{~nm}, \mathrm{a} \sim 10 \mathrm{meV}$ energy-shift is expected when the electric field strength is about $20 \mathrm{kV} / \mathrm{cm}$. The binding energy of the exciton, on the other hand, is estimated to be less than $4 \mathrm{meV}$. Therefore, the QCSE always causes a red-shift in exciton energy. The oscillator strength, on the other hand, is found to decrease as the field increases.

\section{Electrical Field Effect on Surface Phonons}

As recently reported by us, ${ }^{2}$ the use of far infrared reflectance (FIR) spectroscopy to study surface phonons has been proven to be a simple, but very effective and non-destructive technique to identify semiconductor materials in low dimension. It becomes a very powerful analytical tool when certain kind of chemical synthesis is involved. In fact, we have successfully used FIR technique to identify the II-VI and III-V quantum dots formed in hosts by sequential ion implantation and thermal annealing.

That surface phonon comes about when the relevant electromagnetic theory has been applied to solid state physics aspects with appropriate boundary conditions. The dielectric constant $\varepsilon(\omega)$ contains information on the long wave elementary excitations of the solid to which long wavelength electromagnetic fields are related. At the frequency between the TO and LO modes of crystal, the dielectric constant $\varepsilon(\omega)$ is negative and it is where that the surface mode can occur. The electromagnetic field associated with the surface mode are located near the surface. When the particle size gets much smaller that the wavelength of the incident light, which is the case in the present study, only the first order term is considered and it is called Froehlich mode $(l=1)$. In addition, it has been demonstrated ${ }^{10}$ that the observed surface phonon modes are sensitive to the particle size and its dielectric constant of the surrounding matrix.

Due to the limitation of the fabrication processes, it is hard and sometimes impossible to fabricate well-shaped and well-dispersed quantum dots in the host. Therefore, It is very important to develop a particle size and shape dependent characterization technique. It is believed that the study of electric field dependent effects on surface phonons may serve the needs since the local electric field is sensitive to the crystal size and shape. Under certain experimental conditions, different modes with different lirequencies can be observed.

Therefore, our intention in the present effort is to find out the electric field dependency of both exciton energy and surface phonon modes and to understand the fundamental underlying mechanisms which lead to the nonlinear change of the optical properties.

\section{EXPERIMENTAL}

GaAs and CdSe nanocrystals in sapphire were used in the present research. Both GaAs and $\mathrm{CdSe}$ nanocrystals were fabricated via sequential ion implantation followed by thermal annealing. The detailed fabrication and structural analysis have been reported elsewhere. ${ }^{2.3} \mathrm{X}$-ray diffraction ${ }^{2}$ measurements suggest that GaAs nanocrystals in sapphire have particle size in the range of $20-40$ $\mathrm{nm}$ and the crystal structure is found to be cubic zincblende structure. In addition, the lattice constant of the GaAs nanocrystals in sapphire fell in the range of $5.630-5.647 \AA$ which is about $0.1-0.4 \%$ smaller than the bulk value of $5.654 \AA$. " For CdSe nanocrystals in sapphire, the average particle size is about $44 \mathrm{~nm}$. Both hexagonal wurtzite and cubic zincblende crystal structures of CdSe were found in the sapphire substrate with hexagonal wurtzite structure dominant.

The study of the Quantum-confined Stark effect (QCSE) of GaAs and CdSe nanocrystals in sapphire was made possible with Hitachi 3501 spectrophotometer in transmission mode. The spectral 
resolution of $0.2 \mathrm{~nm}$ was used in the present measurements at 320 $1000 \mathrm{~nm}$ region. As illustrated in fig. 1, the electric field was applied by sandwiching the sample between two Nickel mesh. The opening of the grid is $\sim 0.25 \times 0.25 \mathrm{~mm}^{2}$. The wire diameter is $\sim 0.025 \mathrm{~mm}$. The electric field was applied parallel to the light propagation direction, i.e., $E \| k$. The strength of the field was varied from $0-25 \mathrm{kV} / \mathrm{cm}$.

The electric field effect on surface phonon modes of GaAs and $\mathrm{CdSe}$ nanocrystals in sapphire was investigated with BOMEM MB102 FTIR spectrometer with spectral resolution of $1 \mathrm{~cm}^{-1}$ under ambient conditions. Typically 500 scans were used to collect both background and sample spectra. The final reflectance spectra were obtained by taking ratio of virgin substrate against QDs embedded substrate. The applied electric field is perpendicular to the sample surface and the incident angle was $60^{\circ}$.

\section{RESULTS AND DISCUSSION}

Fig. 2 \& 3 show the measured optical absorption spectra of GaAs CdSe nanocrystals embedded in a sapphire substrate in $300-750 \mathrm{~nm}$ spectral region as the function of electrical field. The GaAs nanocrystals in sapphire failed to show any spectral shift under different electric ficlds. For CdSe nanocrystals, an indication of a spectral red-shift is suggested. However, no quantitative information can be obtained without a detailed analysis. Fig. 4 illustrates the electric field dependent far infrared spectra of GaAs nanocrystals in a sapphire host. Under the electric field, a shoulder at higher frequency has been observed. This frequency is further blue-shifted as the electric field increases.

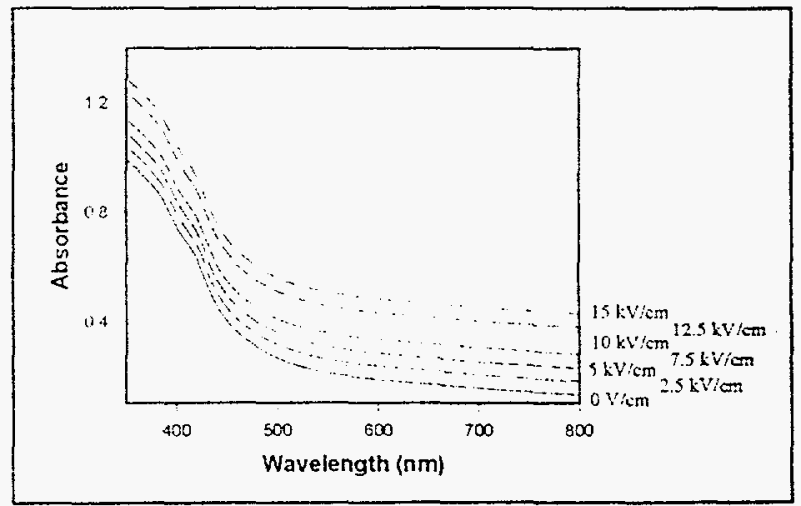

Fig. 2 Optical absorption spectra of GaAs nanocrystals embedded in sapphire matrix as the function of the applied electric filed.

\section{Quantum-Confined Stark Effects on GaAs and CdSe Nanocrystals}

Although quantum-confined stark effect in a quantum-well structure has attracted great attention, a few studies of QCSE on quantum dots have been also reported recently. ${ }^{4.7}$ Like in the case of QWs, when the quantum dots are subjected to static electric field, modification of the total Hamiltonian for an exciton can be evaluated in three terms, i.e. single-particle Hamiltonian for the electron and the hole and Coulomb potential for electron - hole interaction. The measured stark shift can not only be related to the electric field inside the dots, but also be related to the physical size and the shape of the dots. It needs to pointed out that the shape of the dots, on the one hand, affects the degree of the confinement in different directions. On the other hand, it can also modify the effective electric field 
strength inside the dots. ${ }^{8}$ In order to obtain more detailed information on how the exciton energy shifts under an electric field, we have undertaken a series of spectral analyses which can be summarized as follows: 1) use of the first derivative of the original spectra and a curve fitting technique to identify the band edge shift for CdSe QDs in sapphire; 2) combination of the second derivative and curvefitting technique to monitor the band-shift for GaAs QDs in sapphire. Our results shown, as illustrated in fig.5, that the exciton energy of the GaAs QDs was insensitive to the applied field, which may be attributed to number of reasons. i) Excitons present in GaAs QDs may experience strong quantum confinement effects since the estimated exciton Bohr radius $\left(a_{13}\right)$ is about

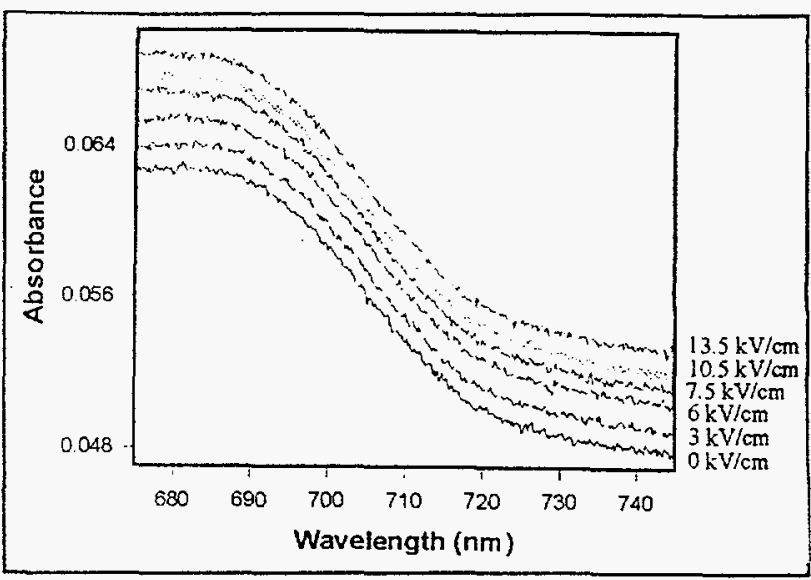

Fig. 3 Optical absorption spectra of CdSe nanocrystals confined sapphire substrate as the function of the applied electric field.

$12.4 \mathrm{~nm}$ which is larger than the average radius of GaAs nanocrystals in sapphire. Therefore, the density of the exciton energy state is increased with respected to the bulk. The Pauli exclusion principle can only limit the level of overlapping of wavefunctions for electrons, holes, and excitons. That is, no two particles can occupy the exactly the same quantum states. Therefore, the repulsion force will constrain the particle wavefunction to overlap. The smaller quantum dots should show weaker response to the applied electric field. Therefore, it is not surprising that no noticeable redshift in exciton absorption energy was detected. The theoretical calculation by Jaziri ${ }^{4}$ suggested only a few meV red-shift. On the other hand, the exciton Bohr radius $\left(a_{\mathrm{B}}\right)$ for $\mathrm{CdSe}$ can be estimated to be $5.4 \mathrm{~nm}$, which is much smaller than that of the nanocrystals fabricated in sapphire ( $\mathrm{R} \sim 20 \mathrm{~nm}$ ). It is expected that the excitons in these CdSe nanocrystals experience weak quantum confinement. Therefore, it should be relatively sensitive to electric field. As indicated in fig. 5 , a $2 \mathrm{meV}$ red-shift in exciton absorption energy was indeed observed. The observed red-shift can be qualitatively related to $\mathrm{F}^{2}$, where $\mathrm{F}$ is the applied electric field.

\section{Electric Field Effects on Surface Phonon Modes of GaAs Nanocrystals}

It is known that when the physical size of the a system reduces to nanometer scale, many physical, optical properties, and even chemical composition can be modified considerably. Due to the large surface to volume ratio, an appreciable number atoms are located in or near nanocrystal surface. The surface and interfacial perturbations can introduce a large amount of anharnomicity to the lattice vibrations. The complex fabrication procedure may introduce a high concentration of defects and possible impurities. In addition, the lattice constant of the GaAs nanocrystals, although resembles its bulk structure, is 0.1 to $0.4 \%$ smaller than its bulk value, which may also indicate that nanocrystals are under strain. All these can attribute to the degree of anharmonicity of the vibrational characteristics. The other possible feature associated with the applied electric field is that

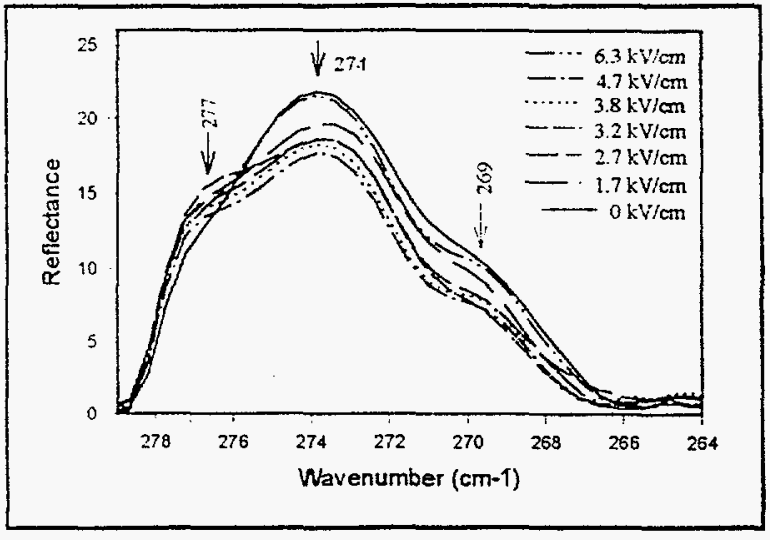

Fig. 4 Far infrared spectra of GaAs nanocrystals in a sapphire substrate as the function of the applied electric field.(note: bulk GaAs $\omega_{\mathrm{To}}=$ $291, \omega_{10}=268 \mathrm{~cm}^{-1}$ ) 
electric field is exerted on these nanocrystals, due to the different dielectric constant of GaAs and $\mathrm{Al}_{2} \mathrm{O}_{3}$, charges will build up at the interface resulting from the polarization. It is also known that the surface modes - behaving like TO modes, are doubly degenerated for cubic crystals. Therefore, it is conceivable that the observed band appearing at $277 \mathrm{~cm}^{-1}$ may be due to the lifting of the degeneracy of the system under the applied electric field.

As mentioned above, there are many factors which can be attributed to the observed anharnomicity of the surface mode. In the following, we may only consider the polarization effect leading to the frequency shifting of the surface mode.

At the absence of electric field $(F=0)$, the potential energy $U_{1}$ of a simple harnomic oscillator can be written as

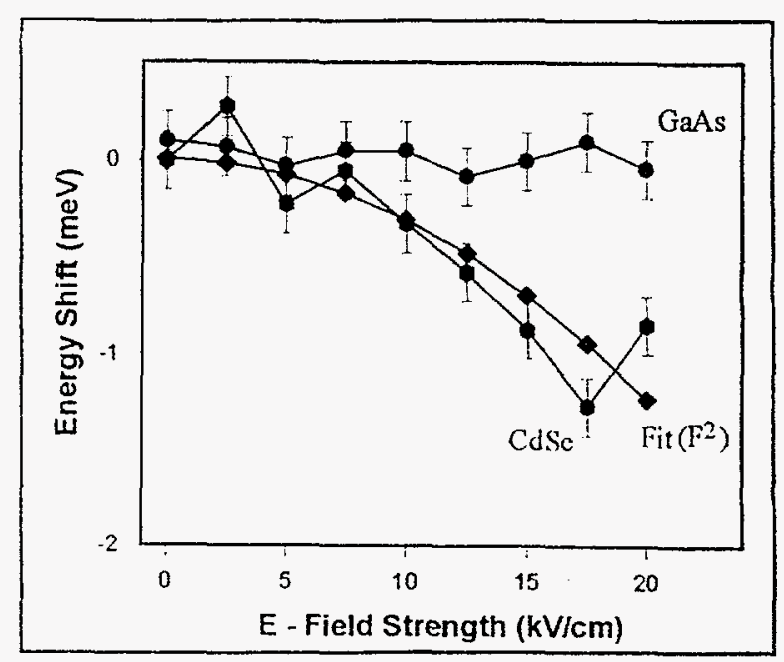

Fig. 5 Exciton energy shift of GaAs and CdSe nanocrystals in sapphire as the function of electric field.

$$
U_{0}(\vec{r}, \vec{F}=0)=\frac{1}{2} \mu \omega_{0}^{2}(\Delta \vec{r})^{2}+U(\vec{r})
$$

where $\omega_{0}$ is oscillator vibrational frequency, $\mu$ is the reduced mass of the system.

$$
\text { When } \mathrm{F}>0 \text {, the potential energy becomes: } \quad U(\vec{r})=U_{0}+\vec{P} . \vec{F}
$$

The second term in equ. (2) is due to the presence of the applied electric field. Since the polarization in the particles is related to the physical properties, shape, interfacial properties, the polarization is not necessarily isotropic. Therefore, the Taylor expansion of the second term can be expressed as follows:

$$
\begin{gathered}
\left.\vec{P} \cdot \vec{F} \propto \sum_{i, j} \chi_{i, j} E_{i} E_{j}\right|_{r-r^{\circ}}+\left.\frac{\partial}{\partial r}\left(\sum_{i, j} \chi_{i, j} F_{i} E_{j}\right)\right|_{r-r \circ} \Delta r+\left.\frac{1}{2} \frac{\partial^{2}}{\partial^{2} r}\left(\sum_{i, j} \chi_{i, j} E_{i} E_{j}\right)\right|_{r-r} \circ(\Delta \vec{r})^{2}+ \\
+(\text { higher order }) \ldots \ldots
\end{gathered}
$$

The first term in equ. (3) is a constant, which will not contributed to frequency change. If the crystal has center symmetry, then the second term is zero. Therefore, the third terms with $(\Delta \mathrm{r})^{2}$ will modify the vibrational frequency. And the frequency shifts is quadratically proportional to the applied field. Unfortunately, due to the complexity nature of the system itself, no attempts are made at the present to analyze the spectra in great detail.

\section{CONCLUSIONS}

We have investigated electric field effects on the exciton energy and surface phonon modes of GaAs and CdSe nanocrystals confined in a sapphire host. Little exciton energy shift was observed for GaAs nanocrystals which may be due to the strong quantum confinement effect. For the excitons weakly confined in CdSe nanocrystals, a $<2 \mathrm{meV}$ red-shift in exciton energy was observed. The relative shift is found to qualitatively proportional to $\mathrm{F}^{2}$.

Electric fields can cause noticeable changes in surface phonon modes of GaAs nanocrystals. The 
anharmonicity and the possible lifting of the surface mode degeneracy are suggested to be the cause of the observed change. This research seems to indicate that FIR spectroscopy is very effective technique to identify nanophase materials in dielectric hosts. When it is coupled with electric field effects, this technique can be further developed into a analytical tool for size and shape characterization.

\section{ACKNOWLEDGEMENTS}

Research is sponsored by the Division of Materials Sciences, U.S. Department of Energy, under contract DE-AC05-84OR2 1400 with Lockheed Martin Energy Systems, Inc. Research at Fisk was performed under grant DE-F605-94ER45521.

\section{REFERENCES}

1. For example, Y. Wang and N. Nerron, J. Phys. Chem. 91, 5005 (1987); O. I. Micic, Z. Li, G. Mills, J. C. Sullivan, and D. Meisel, J. Chem. Phys. 91, 6221 (1987); ibid J. Phys. Chem. 98, 4966 (1994); D. O. Henderson, R. Mu, A. Ueda, A. Burger, K. T. Chen, and D. O. Frazier, Mat. Res. Soc. Proc. 366, 283 (1995); M. Fuji, S. Hayashi and K. Yamakoto, Appl. Phys. Lett 57, 2692 (1990).

2. C. W. White, J. D. Budai, J. G. Zhu, S. P. Withrow, D. M. Hembree, Jr., R. H. Magruder and D. O. Henderson, Mat. Res. Soc. Symp. Proc. 358, 175 (1995); C. W. White, J. D. Budai, J. G. Zhu, S. P. Withrow, D. M. Hembree, Jr., D. O. Henderson, A. Ueda, Y. S. Tung, R. Mu and R. H. Magruder, J. Appl. Phys. (under review); R. Mu, D. O. Henderson, Y. S. Tung, A. Ueda, C. W. White, Jane G. Zhu, JVST B (under review).

3. J. D. Budai, C. W. White, S. P. Withrow, J. G. Zhu, Ion-Solid Interactions for Materials Modification and Processing (Mat. Res. Soc. Symp. fall 1995).

4. S. Jaziri, Solid State Commun. 91, 171 (1994).

5. A. S. Dissanayake, J. Y. Lin, and H. X. Jiang, Phys. Rev. B 51, 5457 (1995).

6. U. Woggon, S. V. Bogdanov, O. Wind, K.-H. Schlaad, H. Pier, C. K. Klingshirn, P. Chatziagorastou and H. P. Fritz, Phys. Rev. B 48, 11979 (1993).

7. Y. Chiba and S. Ohnishi, Phys. Rev. B 38, 12988 (1988).

8. I. Bunget and M. Popescu, Physics of Solid Dielectrics, Chap. 1, pp 15 (Elsevier, 1984).

9. For Example, D. A. B. Miller in Confined Electrons and Photons edited by E. Burstein and C. Weisburch, p 675 - 701 (Nano Series Vol. 340, Plenum Press, New York and London, 1995) (and references therein).

10. R. Ruppin, Electromagnetic Surface Modes edited by A. D. Boardman, chap. 9, p 345 - 398 (John Wiley \& Sons Ltd., 1982); R. Ruppin and R. Englman, Rep. Prog. Phys. 33, 149 (1970); S. Hayashi, Jpn. J. Appl. Phys. 23, 665 (1984).

11. J. S. Blakemore, J. Appl. Phys. 53, R123 (1982) 